
cultures

Les cahiers de l'Acedle

\title{
Mise en œuvre de la recherche en didactique dans un centre universitaire de FLE : démarches, atouts et contraintes.
}

\section{GRASSIN Jean-François et PARPETTE Chantal}

\section{(2) OpenEdition \\ Journals}

Édition électronique

URL : https://journals.openedition.org/rdlc/8157

DOI : $10.4000 /$ rdlc. 8157

ISSN : 1958-5772

Éditeur

ACEDLE

Référence électronique

GRASSIN Jean-François et PARPETTE Chantal, « Mise en œuvre de la recherche en didactique dans un centre universitaire de FLE : démarches, atouts et contraintes. », Recherches en didactique des langues et des cultures [En ligne], 17-3 | 2020, mis en ligne le, consulté le 20 juillet 2021. URL : http:// journals.openedition.org/rdlc/8157; DOI : https://doi.org/10.4000/rdlc.8157

\section{(c) $($ ) $(9)$}

Recherches en didactique des langues et des cultures is licensed under a Creative Commons AttributionNonCommercial-NoDerivatives 4.0 International License 


\section{Mise en œuvre de la recherche en didactique dans un centre universitaire de FLE : démarches, atouts et contraintes.}

\section{GRASSIN Jean-François}

Université Lumière - Lyon 2, Laboratoire ICAR (UMR 5191), France

Jean-François Grassin est Maître de conférences au CIEF à l'Université Lumière Lyon 2 et membre du laboratoire ICAR. Ses travaux s'ancrent dans le champ de la didactique des langues et de l'apprentissage médié par les technologies. Ils s'orientent vers des problématiques liées à l'apprentissage et à l'enseignement du FLE.

jean-francois.grassin[at]univ-lyon2.fr

\section{PARPETTE Chantal}

Université Lumière - Lyon 2, Laboratoire ICAR (UMR 5191), France

Chantal Parpette est Maitresse de conférences honoraire en didactique du FLE à l'Université Lumière Lyon 2 et membre du laboratoire ICAR. Elle travaille dans le champ du français sur objectif spécifique et de la didactique de l'oral. Elle a publié des ouvrages pour les apprenants et pour les enseignants.

chantal.parpette[at]univ-lyon2.fr

Résumé

Dans cet article, les deux auteurs reviennent sur des projets dans lesquels ils se sont investis tour-à-tour en tant qu'enseignant de FLE et enseignant-chercheur en didactique du FLE pour questionner la mise en ouvre de la recherche en didactique du Français Langue Étrangère dans un Centre Universitaire. Leur objectif est d'étudier les formes que prend cette mise en œuvre, pour déterminer à la fois les atouts et les contraintes de ce type de lieu à l'articulation de deux dynamiques, celle des pratiques de recherche et celle des pratiques d'enseignement. Il s'agit d'une description critique recensant les éléments d'un écosystème institutionnel qui revendique la construction de ponts entre ces deux communautés de pratique. La présentation de trois expériences apporte des éléments pour clarifier la relation entre enseignement du FLE et recherche en didactique du FLE. La relation qu'ils analysent ici - et pour laquelle ils plaident - est celle qui fait de la classe non pas un simple lieu de collecte de données pour le chercheur mais bien un lieu qui, par les obstacles qu'elle oppose au déroulement souhaité de l'apprentissage, produit des interrogations et des sujets de recherche. Ce cheminement crée une jonction forte entre recherche et enseignement et aboutit ainsi plus aisément à des supports d'intervention didactique, à dimension variable, nommés ici objets pédagogiques.

Mots-clés : recherche en didactique, intervention didactique, FLE

\section{Abstract}

In this article, the two authors look back at projects in which they have been involved as teachers of French as a Foreign Language and as teacher-researchers. In a FFL teaching center in Lyon university, they aim to question how research has emerged and to examine the forms that it has taken, while determining both the assets and the constraints of this type of place where two dynamics coexist : that of research practices and that of teaching practices. The critical description identifies the elements of an institutional ecosystem that requires the construction of bridges between these two communities of practice. The three experiences exposed provide elements to clarify the interrelatedness between FFL teaching and FFL teaching research. The relation they analyze here - and for which they advocate - describes 
the classroom not simply as a place of data collection for the researcher, but also as a site where the obstacles to the desired course of learning raise questions and research subjects. Thus the strong link between research and teaching leads more easily to didactic intervention supports, with variable dimensions, supports called here pedagogical objects.

Keywords : didactic research, didactic intervention, French as Foreign Language

\section{Introduction}

Si, comme l'écrit Castellotti (2013 : 90), la raison d'être de la didactique des langues implique une relation directe entre recherche et intervention, cette relation se réalise à travers une diversité d'approches. Celles-ci, selon leur plus ou moins grande proximité avec les terrains de l'enseignement-apprentissage, sont souvent catégorisées sous des termes comme didactique de description $v s$ didactique d'intervention. Nous nous rapprochons pour notre part de la position de Coste pour qui la responsabilité sociale du chercheur en didactique inclut «la responsabilité académique et scientifique (...) qui ne signifie nullement que la didactique s'engage comme en surplomb et dans un mouvement d'application sur la 'voie descendante' de la prescription au terrain mais bien plutôt qu'elle a un rôle d'apport participatif » (2013: 53).

En cherchant à questionner ici la mise en æeuvre de la recherche en didactique du Français Langue Étrangère (dorénavant FLE) dans un lieu dédié à l'enseignement de la langue, à savoir un Centre Universitaire de FLE, notre objectif est d'étudier les formes que prend cette mise en œuvre, pour déterminer à la fois les atouts et les contraintes de ce type de lieu à l'articulation de deux dynamiques, celle des pratiques de recherche et celle des pratiques d'enseignement. Nous examinons, au travers de trois études de cas, comment un centre de FLE fonctionne comme espace de médiation entre recherche et intervention. Il s'agit d'une description réflexive recensant les éléments d'un écosystème institutionnel qui revendique la construction de ponts entre ces deux communautés de pratique.

Dans cet article, nous revenons sur des projets dans lesquels nous nous sommes investis tourà-tour en tant qu'enseignant de FLE et enseignant-chercheur en didactique du FLE. Cet article revient sur des projets passés afin de réfléchir de manière située, aux mécanismes du rapprochement entre nos activités de recherche et nos pratiques d'enseignement auprès des apprenants allophones.

Le terme mise en æuvve désigne en français à la fois le début d'une action, une mise en place, et un aboutissement, une application. Nous allons en effet décrire d'une part comment ont commencé certaines recherches, ce qui les a générées, et d'autre part comment, une fois réalisées, elles ont été concrètement appliquées à travers des outils d'enseignement dans un centre de FLE. Nous allons nous attacher à montrer comment un centre universitaire de FLE est à la fois le lieu d'émergence et de traitement de questions de recherche, et le lieu d'une application située de leurs résultats.

Ces deux processus ne sont pas systématiquement couplés. Si les classes de FLE font souvent l'objet de recherche et de recueil de corpus, la plupart de ces travaux ont pour but d'analyser les classes et les comportements des apprenants pour fournir des outils de compréhension aux différents acteurs concernés (enseignants, formateurs). Mais ils ne s'accompagnent pas nécessairement d'un réinvestissement dans l'intervention didactique. Ici, c'est bien au couplage de ces deux facettes de la mise en ouvre de la recherche que nous nous intéresserons, en prenant pour cadre institutionnel le Centre International d'Études Françaises (CIEF) de l'Université Lumière Lyon 2, pour analyser dans quelle mesure et par quels moyens un lieu comme celui-ci offre les conditions d'une complémentarité entre recherche et pratique. 


\section{Comment la recherche existe et s'organise dans un centre universitaire de FLE.}

Un centre universitaire comme le CIEF fait partie d'un écosystème au sein duquel nous avons repéré ce que nous appellerons ici des espaces de médiation entre recherche et pratique. Nous en avons identifié trois catégories :(1) le CIEF en tant que dispositif universitaire, (2) l'appartenance ou son lien avec des groupes associatifs se revendiquant eux-mêmes comme espaces de médiation entre recherche et pratique, (3) sa contribution à des colloques et des séminaires de laboratoire.

\subsection{Un lieu institutionnalisant les liens entre recherche et pratique}

Le CIEF est un lieu où le lien entre recherche et pratique s'inscrit institutionnellement au travers de postes et de dispositifs. Dans un texte publié dans la revue Le Français Dans Le Monde, bien connue des acteurs du FLE, et qui s'adresse aux enseignants et étudiants de FLE, Huver et Charbonneau (2017) décrivent le profil des enseignants qui y travaillent "entre recherche et intervention ». Outre le fait qu'il soit co-écrit par une enseignante-chercheuse et par une enseignante $\mathrm{PRCE}^{1}$, le texte revendique un lien institutionnel fort entre le centre d'enseignement-apprentissage, le département de didactique du FLE/S et le laboratoire de recherche. $\mathrm{Au} \mathrm{CIEF}$, parmi vingt-cinq enseignants titulaires, quatre sont enseignantschercheurs et deux occupent des fonctions d'ATER. Les enseignants-chercheurs des centres de FLE sont statutairement membres de laboratoires de recherche, lesquels définissent le profil « recherche » de leur poste au moment du recrutement. Cela n'a pas toujours été le cas dans le passé, mais depuis quelques années, le profil recherche des enseignants-chercheurs du CIEF concerne des questions liées aux projets d'innovation pédagogique du centre, notamment en Tice $^{2}$ et en Français sur objectif universitaire, pour les plus récents.

Par ailleurs, l'université met en place des dispositifs d'incitation à la recherche : appels à projets pédagogiques, aménagement de service pour des enseignants doctorants, financement de colloques et de déplacements à des colloques constituent autant de possibilités mises au service des enseignants pour leur permettre de se tourner vers la recherche.

Enfin, le CIEF est également le terrain de travaux de recherche d'étudiants en master de didactique du FLE qu'il accueille en stage.

\subsection{Un lieu s'intégrant à un réseau de centres d'enseignement}

Le CIEF est membre d'un réseau de centres universitaires de FLE, Campus-FLE ADCUEFE. Sur son site $^{3}$, l'association présente les atouts de ces centres en identifiant le lien entre recherche et pédagogie de la manière suivante :

Parce qu'ils sont au sein des Universités françaises, les membres de Campus-FLE ADCUEFE sont directement impliqués dans la recherche en didactique du français langue étrangère et dans l'innovation pédagogique. Ils sont donc les plus compétents pour mettre en cuvre les dernières méthodes d'enseignement élaborées. (CampusFLE ADCUEFE)

Le texte introduit une causalité explicite entre le statut institutionnel de ces centres et le lien entre pratiques d'enseignement et recherche en didactique : le centre, parce qu'universitaire, serait compétent pour mettre en œuvre les résultats de la recherche.

Cette médiation entre recherche et pratique est notamment matériellement et symboliquement construite par l'association à travers l'organisation d'un colloque tous les deux ans. Le dernier s'est déroulé à Montpellier en 2018, organisé par le centre universitaire de FLE et le

\footnotetext{
1 Professeur Certifié affecté dans l'enseignement supérieur

2 Technologies de l'information et de la communication pour l"enseignement

3 https://www.campus-fle.fr/
} 
laboratoire de recherche Praxiling, et avait pour objet la formation de formateurs en langues. Le texte de présentation en appelle aux acteurs et à leurs représentations de la professionnalisation pour reconnaître les liens indispensables recherche/pratique :

Ce colloque s'adresse à tout enseignant ou formateur en langues, présent ou futur, à tout acteur de l'enseignement-apprentissage des langues reconnaissant le rôle central et continu de la formation dans la professionnalisation et désireux de participer à une réflexion commune sur l'art de conjuguer l'expérience professionnelle et sa conceptualisation. Une telle réflexion est indispensable pour développer des dispositifs de formation responsables, capables de répondre aux attentes des enseignants de langues et de les outiller conceptuellement pour répondre aux exigences de contextes professionnels en évolution. (nous soulignons).

Le colloque se veut ainsi un espace de réflexion et de conceptualisation - que nous traduirons par recherche - et qui se donne pour but de (1) développer des dispositifs de formation, et (2) répondre aux attentes enseignantes (3) dans des contextes professionnels en évolution. Le lien entre pratique et recherche se fait au profit d'un certain type de recherche, la rechercheintervention, une recherche oscillant entre science et action (Marcel et Bedin, 2018). Le texte de l'appel à communication parle de « réflexion commune » et s'adresse directement à " tout enseignant ou formateur en langues » et à «tout acteur de l'enseignement-apprentissage des langues » sans différencier explicitement enseignant et enseignant-chercheur. Le discours d'accompagnement du colloque traduit la médiation qu'il se propose d'instaurer entre ces deux espaces d'activités.

\section{Comment la recherche est mise en œuvre dans un centre universitaire de FLE}

Après avoir identifié ces espaces et dispositifs de médiation, qui sont autant d'atouts pour des circulations recherche / pratique, nous examinons de manière plus précise les formes que prennent ces mises en œuvre.

Nous avons repéré des sujets dont la recherche s'est emparée et verrons dans quelle mesure ces sujets ont donné lieu à une circulation du savoir par des produits de la recherche, des objets pédagogiques, et dans des changements de pratiques.

Les objets identifiés dans nos études de cas sont de nature différente. Deux consistent en des manuels de FLE, l'un pour un public spécialisé en économie-gestion, l'autre généraliste. Quant au troisième, il s'agit d'un dispositif médiatique, un réseau social numérique dédié au centre universitaire lui-même.

Pour plus de clarté, le «nous» va laisser ici la place à des «je» rendant compte de l'expérience de chacun des deux auteurs du présent article.

\subsection{Deux manuels conçus de manière située.}

Je vais tenter de retracer le cheminement réflexif suscité par des situations d'enseignement et qui a conduit à la création de deux manuels dans laquelle j’ai été impliquée ${ }^{4}$.

\subsubsection{Réussir ses études d'économie-gestion en français}

$\mathrm{Du}$ fait de l'absence de traces concrètes de cette partie du cheminement, on peut être tenté d'oublier que la recherche en didactique part pour l'essentiel de la classe et que la création d'objets pédagogiques constitue une réponse concrète aux interrogations que celle-ci a générées. Le manuel Réussir ses études d'économie-gestion en français est un ouvrage de Français sur objectif universitaire destiné aux étudiants allophones souhaitant faire des études d'économie dans une université française. Il comporte une trentaine de séquences

\footnotetext{
${ }^{4}$ Il s'agit ici de Chantal Parpette.
} 
pédagogiques de niveau B1-B2 portant sur l'organisation des études d'économie, la compréhension des cours magistraux à partir d'enregistrements de cours authentiques, la lecture de documents d'économie, la méthodologie d'exposé, la méthodologie de la dissertation d'économie.

Cet ouvrage trouve son origine une trentaine d'années plus tôt, dans les années 80 , lorsque des équipes d'enseignants de FLE, dont je faisais partie, exerçaient dans des écoles d'ingénieurs accueillant en formation continue des ingénieurs étrangers de pays en développement, comme l'École Nationale des Travaux Publics de l'État (Lyon), ou encore l'Institut Agronomique Méditerranéen (Montpellier). Nous nous sommes trouvés confrontés à des besoins de formation linguistique que la didactique du FLE n'avait pas encore explorés. Il s'agissait en effet pour ces ingénieurs de niveau peu avancé en français d'être rapidement opérationnels dans leurs cursus d'ingénieurs. Il fallait s'intéresser aux discours disciplinaires des cours de travaux publics ou d'agronomie. Sur le plan matériel, les conditions étaient relativement favorables à la mise en place de la démarche. La formation linguistique étant prise en charge au sein de l'établissement scientifique lui-même, la proximité avec le cursus scientifique ciblé rendait quasi évident le rapprochement des cours de langue avec les cours de science. Par ailleurs, la présence de services audiovisuels largement dédiés à l'enseignement permettait l'enregistrement vidéo de cours, d'activités de laboratoire ou d'interviews de différents acteurs scientifiques de la formation. Nous étions là au cœur de ce que Mangiante et Parpette modéliseraient 25 ans plus tard (2004) sous le terme de « collecte des données » en Français sur objectif spécifique.

Cette démarche empirique d'enseignants n'était pas encore de la recherche. Confrontés aux cours magistraux, nous étions déroutés par leurs caractéristiques discursives éloignées de celles des documents que traitait - et continue de traiter - le français général. La recherche a en effet montré plus tard que les cours magistraux se caractérisaient, entre autres, par leur polyfonctionnalité, leurs implicites liés au vécu partagé entre les enseignants et leur public d'étudiants, leurs faits de dialogisme, spécificités absentes des discours proposés dans les méthodes de français général (voir Bouchet et Parpette, 2017 ; Dufour et Parpette, 2017 et 2018). A cette époque, nous n'avions aucune stratégie de traitement de ces phénomènes langagiers en FLE au point que cela nous a conduits à des erreurs dans l'élaboration des séquences de compréhension orale de cours magistraux et qu'il nous est arrivé de renoncer à travailler sur ces enregistrements de cours faute de savoir comment les traiter. A ces enregistrements authentiques de cours, nous avons préféré des documentaires scientifiques, qui, malgré leur complexité notionnelle, avaient l'avantage d'avoir la construction discursive linéaire et objectivée des écrits oralisés et non les spécificités des discours oraux en face-àface. Nous avions des outils didactiques pour traiter les premiers mais pas les seconds.

Ce contexte d'enseignement, avec ses interrogations et ses solutions empiriques - ou souvent, absence de solution - a donné naissance, une bonne dizaine d'années plus tard, à un travail de recherche mené au sein du laboratoire que j'avais intégré au moment de ma nomination comme enseignante-chercheuse à l'université. Le séminaire consacré à cette réflexion s'intitulait « Initiative campus » (INCA), du nom d'un appel à projets de la Région RhôneAlpes dédié à l'amélioration de l'enseignement universitaire. C'est au sein de ce séminaire qu'ont été menées les premières analyses discursives de cours magistraux issus de l'École des travaux publics (Bouchard, Parpette et Pochard, 2005 ; Bouchard et Parpette, 2012) et qu'ont été mises au point les premières procédures d'enseignement de ces discours en FLE, de manière encore assez limitée.

L'ouverture au CIEF de Lyon 2 d'un DU « FLE-Économie » comportant bien évidemment des cours de FLE préparatoires au cursus d'économie, a ainsi constitué une jonction entre la recherche et l'intervention didactique. Nous avons repris l'enregistrement de cours magistraux, en économie cette fois, pour, simultanément, poursuivre l'analyse de discours de 
manière très ciblée et constituer des séquences pédagogiques qui sont devenues quelques années plus tard un matériel édité complet Réussir ses études d'économie-gestion en français (Parpette et Stauber, 2014). Ce matériel sert actuellement d'appui aux formations FOUÉconomie (Français sur objectif universitaire-Économie).

Le schéma ci-dessous retrace le cheminement qui vient d'être décrit.

Figure 1 : La co-construction d'un objet pédagogique et de recherche : l'exemple d'un dispositif FOU

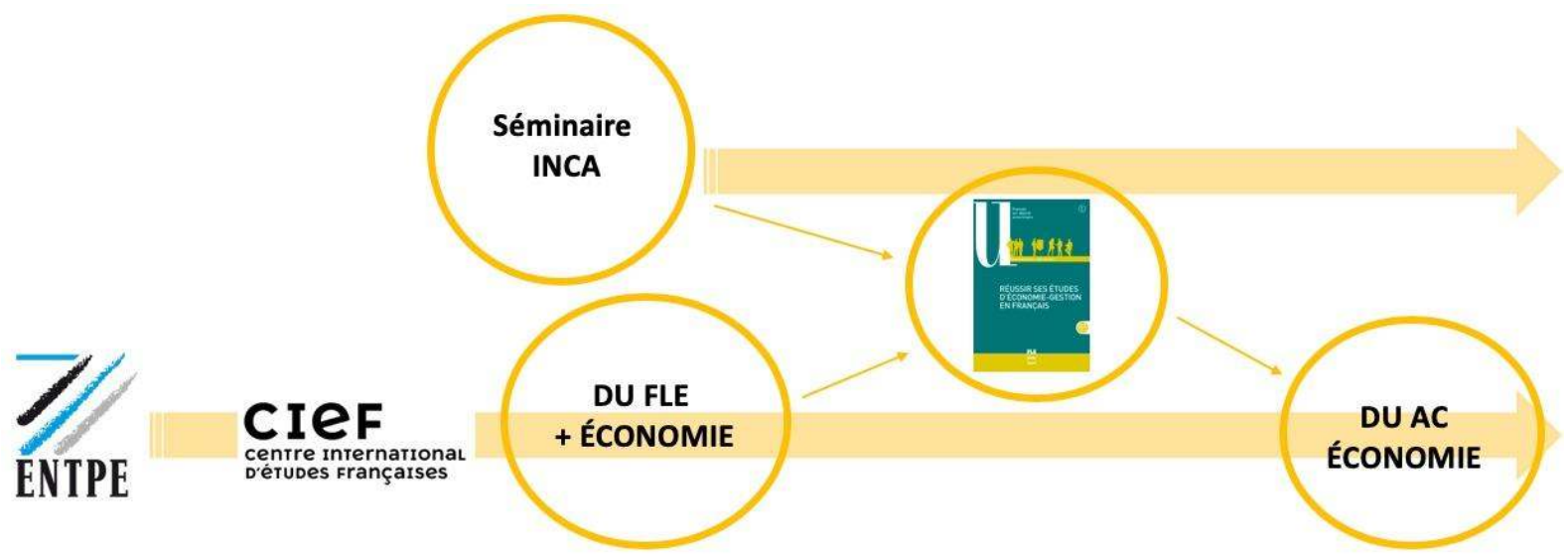

Ainsi, depuis les premières interrogations des enseignants de FLE confrontés aux nouveaux besoins de publics étudiants, l'émergence de travaux de recherche qui en a résulté dans le contexte - favorable - d'un centre universitaire de FLE, jusqu'à la réalisation et la mise en œuvre de séquences pédagogiques issues de ces questions de recherche, les interrogations de départ trouvaient leur réponse dans le travail combiné de la recherche et de la création d'outils d'enseignement du FLE. Cet aboutissement a servi de modèle à d'autres réalisations de matériel en français sur objectif universitaire, notamment à travers la création d'une collection FOU aux Presses universitaires de Grenoble, qui compte à ce jour quatre ouvrages consacrés à des disciplines universitaires.

\subsubsection{ICI, une méthode de français général}

Une relation similaire entre cours de langue et recherche s'est trouvée à l'œuvre autour de la notion $d$ ' " enseignement en milieu homoglotte ». Le milieu environnant francophone a toujours été partiellement pris en compte dans les centres de FLE en France, à travers des visites, des enquêtes, ou des spectacles, à travers également l'accès facile à la presse quotidienne, écrite ou télévisuelle. Mais cette relation avec le milieu environnant paraissait assez marginale au regard du potentiel que représente un tel contexte pour l'apprentissage de la langue, et interrogeait certains enseignants au point que l'ADCUEFE s'est saisie de cette question lors d'un colloque en 2005 intitulé L'enseignement-apprentissage du FLE en milieu homoglotte : spécificités et exigences. Cette rencontre a stimulé la réflexion et j'ai présenté une communication qui proposait un programme de cours intégrant des aller-retours systématiques entre la classe et le milieu, le milieu offrant à la fois des situations d'application des acquis de la classe et d'acquisition de nouveaux savoirs, à travers des activités d'observation de comportements langagiers en situations naturelles, et des tâches interactionnelles avec des acteurs extérieurs.

Cette réflexion a abouti à la création d'une méthode FLE, intitulée Ici (Abry et al., 2006), classique dans sa partie concernant l'apprentissage en classe, mais innovante pour les séquences extérieures qui accompagnaient chacune des unités de la méthode. Ces activités extérieures, nommées Découvertes, sont au nombre de 5 ou 6 et réalisées à la fin de chaque unité par les apprenants. Au début de l'apprentissage, les découvertes consistent à observer et noter des comportements langagiers. Ainsi, par exemple, à l'issue de l'unité sur les échanges 
dans les commerces, les apprenants partent par deux dans des marchés, ou s'installent à la terrasse d'un café pour repérer les interactions, les énoncés utilisés, les manières de faire. Après l'unité sur les études et le travail, ils entrent en contact avec des étudiants ou des professionnels pour les interroger sur leurs activités, leur emploi du temps. Une fiche de consignes organise ces découvertes, amenant les apprenants à noter les informations recueillies mais aussi les expressions nouvelles, leurs impressions face aux situations, leurs interrogations, etc. Toutes ces données font ensuite l'objet d'un moment de partage et de discussion dans la classe.

La méthode Ici une fois éditée a été utilisée, comme les autres méthodes, dans un certain nombre de centres de FLE. Elle a été adoptée pendant plusieurs années avec certains groupes du CIEF pendant qu'une des auteures conduisait dans le même temps une recherche doctorale sur ce dispositif d'apprentissage combiné entre classe et milieu naturel. Les années passées rendant obsolètes certaines données, Ici a été abandonnée en tant que méthode support de cours mais le concept est resté actif, et l'actuelle directrice des études envisage de reconstruire la démarche autour d'autres méthodes de FLE. Reconstruire la démarche ne signifie pas reprendre les activités à l'identique mais garder le principe de l'organisation d'un apprentissage combiné entre classe et milieu extérieur, cette combinaison pouvant prendre des formes diverses.

Figure 2 : La co-construction d'un objet pédagogique et de recherche : l'exemple d'un manuel de découverte du milieu homoglotte

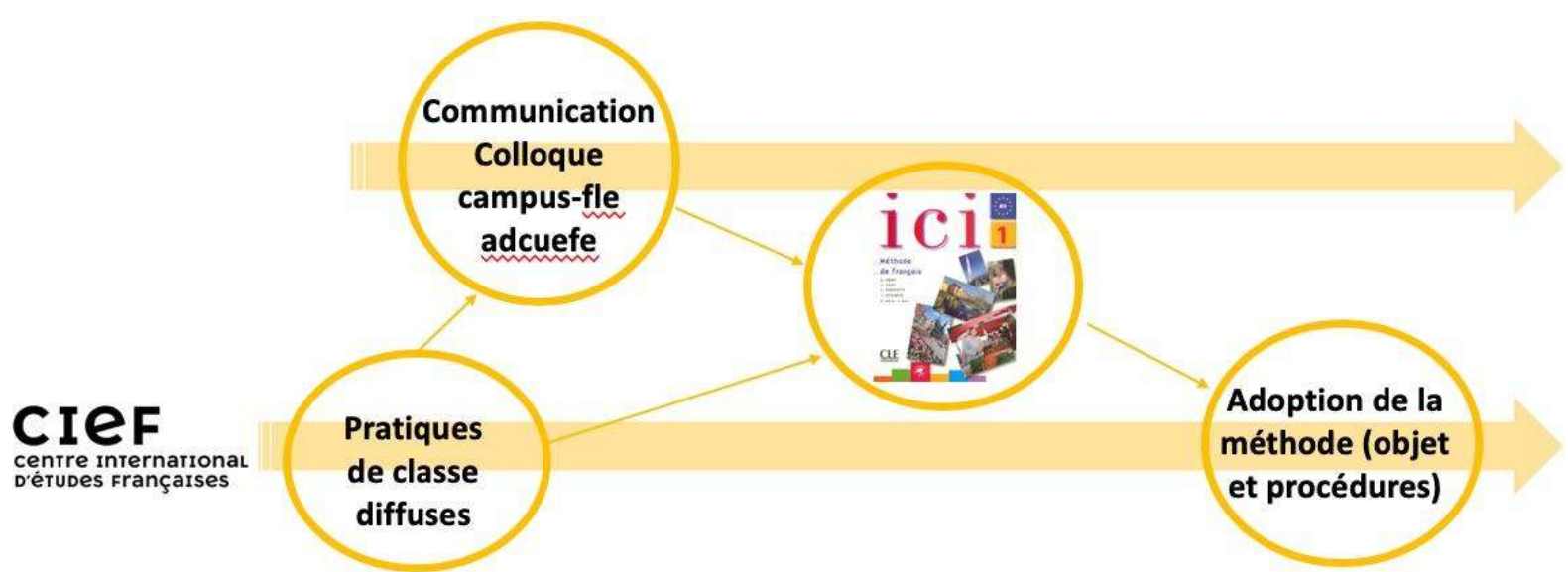

Comme le montrent le schéma ci-dessus (figure 2) et le précédent (figure 1), la combinaison entre les interrogations suscitées par la classe et le travail de recherche a suivi un cheminement aboutissant à une transformation sur le terrain de la classe et de ses pratiques, par la production d'un outil pédagogique tentant de répondre aux questions initiales.

\subsection{Un dispositif médiatique pour l'enseignement-apprentissage : le cas d'un réseau social.}

Le troisième objet construit dans cette interface entre pratique de recherche et pratique d'enseignement-apprentissage et qui nous permet de décrire les rapports de ces pratiques est la mise en place d'un réseau social numérique destiné à accompagner les formations en Français Langue Étrangère de l'institution.

Cet objet pédagogique, le Rezo Lumière, est un dispositif médiatique combinant les fonctionnalités des réseaux sociaux numériques (page et profils individuels, statut, fil d'actualité) et des outils de travail et d'interaction collectifs (groupes et forums notamment) ${ }^{5}$

\footnotetext{
5 Pour une description plus complète, voir Grassin, 2013.
} 
permettant des interactions numériques natives ${ }^{6}$ et la publication d'activités pédagogiques liées au contexte de classe. Des activités pédagogiques ont été élaborées soit dans le cadre de modules tutorés d'auto-apprentissage (de type actionnel comme se trouver une colocation), soit dans le cadre de cours en présentiel. Cet environnement permettait de faciliter les échanges pédagogiques à l'intérieur des groupes-classes mais aussi aux étudiants de communiquer entre eux, au sein d'une communauté d'apprentissage institutionnelle, incluant leurs pairs et les enseignants.

Arrivé deux ans auparavant au CIEF, j'ai conçu cet objet et l'ai proposé à la communauté à l'époque où j'étais chargé de mission TICE au CIEF, en $2009^{7}$. L'enjeu pédagogique était d'intégrer d'autres situations de communication aux situations de classe en présentiel paraissant propices à un apprentissage de la langue et à une socialisation langagière dépassant le groupe-classe, afin d'enrichir le répertoire communicatif des étudiants et leurs opportunités communicatives.

La décision d'en faire un objet de recherche a été prise environ un an plus tard et soutenue ensuite par l'institution et l'obtention d'un aménagement de service en vue d'un travail de thèse, débuté en 2010 et soutenu en 2015. Pendant dix ans (la plateforme de réseau social numérique a été fermée en 2019), le dispositif médiatique a permis la mise en place d'activités pédagogiques pour les cours en présentiel de l'institution, de développer des activités en ligne les accompagnant, mais aussi de créer en un même lieu, des espaces d'interaction en ligne plus ou moins formels (blogues, forums, réseau social), individuels et collectifs.

La question qui se posait était de savoir pourquoi les interactions en ligne ne faisaient pas partie de manière institutionnelle des répertoires pédagogiques des enseignants et des situations discursives d'action des étudiants, si ce n'est sous la forme de simulations sur papier. Des entretiens m'ont permis de recueillir des représentations de ces usages et la manière dont ceux-ci pouvaient ou non s'intégrer à un répertoire de pratiques professionnelles. Je renvoie aux différents articles publiés pendant ce travail doctoral (par exemple Grassin 2017a, 2017b) et je focaliserai ici mon propos sur la question des limites du rapprochement des intérêts des deux sphères sociales de la pratique et de la recherche.

Sur un terrain où les pratiques se réfèrent à la classe et au milieu homoglotte comme contextes d'apprentissage à l'exclusion des espaces discursifs et interactionnels en ligne, mon objectif était d'échapper à une posture militante à l'égard de l'intégration de l'environnement socionumérique comme contexte d'apprentissage que me renvoyait ma position de chargé de mission numérique dans l'institution, pour adopter une posture compréhensive tout en gardant des objectifs praxéologiques. Le travail doctoral était une tentative d'interroger des pratiques, des croyances et des représentations par la recherche, en créant une "zone interprétative » (Desgagné et al., 2001 : 37), autour de la pratique comme objet d'exploration. Le but de cette exploration se construisait entre deux pôles, celui guidé par la pratique et le développement de répertoires nouveaux - concernant ici les interactions d'apprentissage, en ligne et en réseau « en vue d'aider les enseignants à composer avec des situations inédites de la pratique » (ibid. : 38), et celui guidé par la recherche, plus conceptuel.

La recherche se fixait à la fois l'objectif d'une meilleure compréhension du fait interactionnel en ligne dans un cadre pédagogique en proposant une catégorisation des formes relationnelles et interactionnelles à l'œuvre sur un réseau pédagogique en langue, et celui de pistes didactiques pour la configuration d'activités pédagogiques. Il s'agissait de construire une pensée pédagogique du réseau autour d'affordances sociales et communicatives, afin que les enseignants puissent y trouver leur place et se saisir de ces nouveaux contextes interactionnels

\footnotetext{
6 C’est-à-dire écrites sur le site pour être lues sur le site (cf. Paveau, 2017).

7 Il s'agit ici de Jean-François Grassin.
} 
de manière adaptée, dans une meilleure compréhension des littératies numériques et des genres communicationnels.

Cependant, cette démarche réflexive était avant tout individuelle et mon objet de recherche ne se trouvait pas au cœur des pratiques de la communauté de praticiens qui m'intéressait, puisque les usages réseau socio-pédagogique étaient des usages en périphérie des pratiques enseignantes au CIEF, des pratiques sur « un mode mineur ».

Dans ce contexte, quels sont les résultats d'une telle recherche et son réinvestissement dans les pratiques locales? Le travail de recherche a permis d'accompagner une appropriation pédagogique par les enseignants d'un nouvel environnement de travail, reconnu comme possibilité d'actions pédagogiques mais restant toutefois à la marge des pratiques les plus partagées. Le questionnement de la recherche autour des littératies numériques a, de plus, amené la direction pédagogique à inscrire dans les curricula de l'institution certaines pratiques numériques d'écriture (participation à des forums, activités de tchat...). Une étude serait à mener sur les effets dans les classes d'une telle inscription.

Figure 3 : La co-construction d'un objet pédagogique et de recherche : l'exemple d'une plateforme sociale

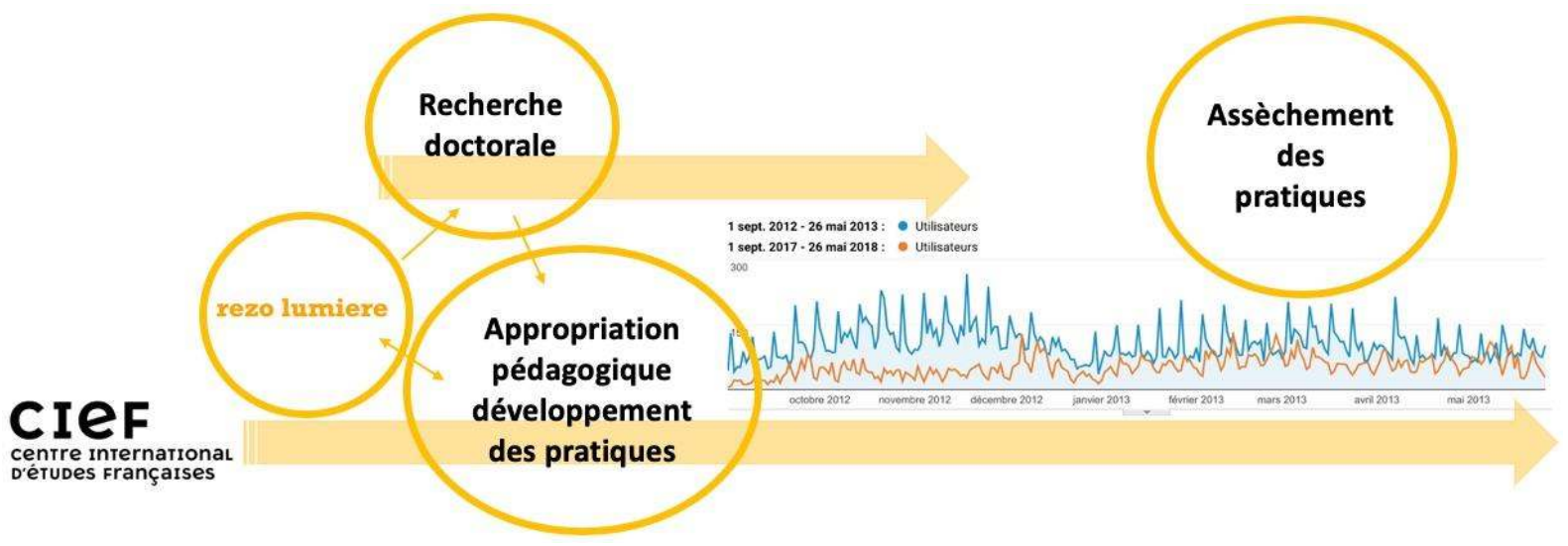

Il était pour moi intéressant de revenir, quelques années après sur ce qui se passait sur ce réseau en ligne et voir si les pratiques avaient évolué. L'observation des traces d'activités par un outil comme Google analytics a permis de constater de manière quantitative des usages en diminution constante 8 . De même, le suivi des usages de manière qualitative montre un assèchement des pratiques vers de moins en moins de saisies des affordances conversationnelles du réseau : le site sert avant tout, trois ans après la fin de la recherche, à la mise en ligne de productions d'étudiants effectuées hors de la plateforme (il n'y a pas ou peu de productions natives) ou à la participation à des forums.

Alors que la plasticité interprétative que je donnais au réseau pédagogique me semblait être un terreau favorable à des pratiques exploratoires, quelques années après ce travail, force est de constater qu'il y a eu des appropriations individuelles mais pas d'appropriation explicitement collective. Alors qu'il s'agissait d'en faire institutionnellement un « laboratoire de pratiques » (Rivens-Mompean, 2013 : 335), l'appropriation s'est faite au niveau individuel, au travers de logiques ponctuelles, opportunistes et utilitaristes, sans doute notamment car aucun collectif de praticiens n'a été mobilisé par le travail de recherche mais seulement des utilisateurs individuels. Ne suffisant pas à les installer dans une culture d'établissement, cette plasticité est à la fois un atout, pour initier des usages, mais également un inconvénient quand il s'agit d'instituer des pratiques.

\footnotetext{
${ }^{8}$ Dans la figure 3, la ligne haute du graphique représente l'activité du site de manière quantitative pendant la recherche doctorale, la ligne basse celle des années suivant cette recherche.
} 
Ce retour réflexif fait entrevoir deux points :

- Le terrain de l'institution permet de créer des objets tels que ce dispositif pédagogique, et le couplage avec un objet de recherche permet un travail de discussion autour de pratiques de terrain. Des résultats ont été montrés lors de journées pédagogiques organisées par l'institution et, même si la conduite de la recherche n'avait pas prévu de mobiliser les praticiens dans la réflexion et l'élaboration des questionnements autour de l'objet pédagogique, la période où elle a eu lieu correspond également à un temps d'expérimentations pédagogiques impliquant un grand nombre d'acteurs. Sur le terrain, cette plateforme pédagogique a été objet de discussions et de négociations (de " traductions » selon Akrich, 2006) en amenant les acteurs de l'institution à se positionner sur des savoirs tels que les interactions pour l'apprentissage, les communautés de réseau, en questionnant ses pratiques.

Pour autant, Perrenoud rappelle qu'il « arrive bien évidemment à chacun de réfléchir spontanément sur sa pratique, mais si cette mise en question n'est ni méthodique, ni régulière, elle ne mène pas nécessairement à des prises de conscience et à des changements » (Perrenoud, 2001: 42). La recherche n'échappe pas à une perception « top down », alors même que la méthodologie de recherche se voulait au plus près des représentations et des pratiques des acteurs dans une perspective émique (Olivier de Sardan, 2008), avec la volonté de fournir un autre cadre interprétatif de ces pratiques nouvelles permettant de réfléchir ensemble autour de concepts mobilisateurs, tels que les formes de conversation en ligne et les effets des réseaux numériques sur l'engagement et la production des apprenants. L'objet pédagogique est apparu comme avant tout technocentré et les enjeux en termes d'apprentissage n'ont pas suffisamment été l'objet de traductions ou d'actions des enseignants pour lui donner une dimension collective. Or, les usages des objets pédagogiques ne tiennent pas uniquement à leurs propriétés intrinsèques mais à leur capacité de fédérer un large réseau d'acteurs.

\section{Discussion : limites et leviers}

La présentation de ces trois expériences apporte des éléments pour clarifier la relation entre enseignement du FLE et recherche en didactique du FLE. La relation que nous analysons ici et pour laquelle nous plaidons - est celle qui fait de la classe non pas un simple lieu de collecte de données pour le chercheur mais bien un lieu qui, par les obstacles qu'elle oppose au déroulement souhaité de l'apprentissage, produit des interrogations et des sujets de recherche. Ce cheminement crée une jonction forte entre recherche et enseignement et aboutit ainsi plus aisément à des supports d'intervention didactique, à dimension variable, ce que nous appelons ici objets pédagogiques. Comme nous l'avons évoqué au début de l'article, le statut du lieu d'intervention analysé, à savoir un centre universitaire d'enseignement du FLE, joue un rôle tout à fait déterminant pour les raisons déjà mentionnées. Cela n'exclut cependant pas les obstacles à cette jonction que nous souhaitons entre recherche et enseignement aux publics allophones. C'est ce que nous proposons d'examiner dans cette dernière partie afin de comprendre pourquoi celle-ci est loin de se réaliser systématiquement.

\subsection{Limites}

La première limite tient au fait que rassembler dans un même lieu institutionnel des praticiens et des chercheurs-praticiens n'implique pas l'évidence d'une recherche située empirique et/ou interventionniste. Les espaces d'intéressement ne sont souvent pas les mêmes entre chercheurs et praticiens et même une recherche à dimension fortement interventionniste ne se fixe pas des objectifs temporels aussi rapprochés que ceux qu'implique un projet pédagogique.

Selon les cas, d'autres limites peuvent apparaître. Ainsi pour la mise en œuvre, dans les cours, de la méthode $I c i$, les auteurs-utilisateurs se sont heurtés aux représentations de la direction du 
CIEF qui ne savait pas comment envisager les activités extérieures à l'espace-classe. Fallait-il les considérer vraiment comme des moments d'apprentissage à part entière ? La crainte était que les apprenants, qui sont aussi des clients, ne prennent pas au sérieux ces activités. Les enseignants n'ont donc pas pu obtenir que les heures consacrées aux découvertes soient intégrées dans les emplois du temps, et ces activités ont dû être proposées aux apprenants endehors des heures officielles de cours. Ce qui posait le problème inverse, à savoir que certains apprenants étaient réticents à réaliser ces activités qui venaient s'ajouter à leur emploi du temps. Lorsque ces réticences étaient partagées par un nombre conséquent d'apprenants, cela ruinait à l'évidence le projet pédagogique de la méthode. S'ajoutait à cela, la difficulté liée à la rémunération de ces activités. Les centres de langues qui ont l'habitude de rémunérer les enseignants en fonction du nombre d'heures de cours en présentiel, ont du mal à imaginer un modèle de rémunération pour des formes d'enseignement-apprentissage hybrides entre classe où l'enseignant est présent, et hors-classe où les apprenants sont seuls. Cette remarque vaut $a$ fortiori pour tout ce qui est activité de conception et de tutorat en ligne, dans un établissement où la culture d'enseignement/apprentissage est présentielle. On comprend alors la difficulté supplémentaire à faire entrer l'activité de recherche dans le temps de travail d'une équipe enseignante où la majorité n'est rémunérée qu'en rapport à son temps d'enseignement.

Une dernière limite serait le fait que ces recherches, si elles émanent d'interrogations produites par le terrain et si elles peuvent avoir une action transformative, ne débouchent pas sur des « mises en recherche » collective d'équipes de praticiens. Le projet de recherche reste souvent à l'aune de l'individu et les formes de conceptualisation qui en émanent sont peu reprises collectivement.

\subsection{Leviers}

Si ces expériences de couplage entre recherche et conception pédagogique comportent des limites, elles nous montrent pourtant des pistes réelles allant vers la co-construction d'objets pédagogiques. Il est important d'envisager des espaces réflexifs (groupes de travail ou séminaires de recherche) par lesquels le praticien comme le chercheur peuvent interroger les pratiques individuelles en tant que participation à une communauté de pratiques, afin que les buts praxéologiques de la réflexion aboutissent, selon les objectifs fixés des deux côtés, à une conceptualisation de la pratique et son amélioration, son évolution ou son changement.

Un premier espace où les deux sphères de pratiques peuvent négocier des objets pédagogiques peut être les journées pédagogiques du CIEF qui ont lieu une à deux fois par an (en janvier et en juin). Pendant ces journées, adressées à la communauté enseignante du CIEF, un temps est prévu pour rendre compte de recherches didactiques qui avaient pour terrain le CIEF. Chaque année, deux ou trois étudiants de master FLES sont en stage dans l'institution et construisent une problématique de recherche liée à ce stage et à la conception pédagogique. Il s'agit de bonnes opportunités pour montrer comment la recherche et la pratique s'alimentent mutuellement. Par contre, ce sont des moments discursifs du type « que dit la recherche ? » ou de " retour vers l'enquêté », lors desquels le praticien peut se retrouver dans la position d'un interlocuteur passif. Ces temps de compte-rendu méritent d'être plus formalisés de façon à déboucher sur des prolongements, dans l'une ou l'autre des sphères de pratique : comment sur le terrain s'empare-t-on des résultats de la recherche ? Quel objet pédagogique en commun conçoit-on ? Les intérêts pour la communauté de pratique et celles pour la communauté de recherche (cf. Desgagné, 1997) doivent être exposés en parallèle.

Un autre espace d'intéressement pourrait être la conception d'un séminaire de recherche au sein même du CIEF, mené par des enseignants-chercheurs (ils sont six, quatre MCF et deux ATER) et ouverts aux enseignants. Ce séminaire permettrait de recenser les recherches qui ont pour terrain le CIEF et de faire émerger des thèmes de recherche problématisant les questions rencontrées par les acteurs dans leur pratique. 
Ces deux espaces fonctionnant en parallèle permettraient sans doute de mieux prendre en compte des " logiques d'intéressement » (Akrich et al., 1991) de façon à ce que chacun, enseignant-praticien ou enseignant-chercheur, trouve dans l'objet pédagogique des réponses possibles à des besoins, des attentes ou des projets. Ils pourraient construire une pratique réflexive qui aurait moins à voir avec la didactique appliquée, c'est-à-dire une démarche cherchant à appliquer des solutions issues de la recherche au risque que les acteurs s'y engagent faiblement, et plus avec une didactique située, une "science de l'agir professionnel » (Schön, 1993/1983). Dans ce cadre, deux directions pourraient être prioritaires : l'identification de besoins de formation et l'ouverture de nouvelles offres de formation, notamment prenant mieux en compte le numérique comme partie intégrante de l'écologie d'apprentissage. Or, dans les trois exemples que nous avons recueillis dans ce texte, l'amorçage de ces liens entre recherche et pratique est peu le fait de l'institution mais plus souvent d'individus.

Les projets pédagogiques doivent également pouvoir inclure des temps réflexifs tournés vers la recherche et financés par l'institution. L'université Lyon 2 ayant récemment décidé d'appuyer le développement de nouveaux projets pédagogiques par des décharges de cours, il serait intéressant que dans ce cadre, un lien avec la recherche puisse se formaliser, par exemple sur le modèle de la PEER (Practice Embedded Educational Research) (Goigoux, 2017).

\section{Conclusion}

Les centres universitaires de FLE sont indiscutablement les lieux où la réflexion didactique peut à la fois voir émerger ses objets de recherche et voir mis en œuvre les résultats qui en découlent. Et ceci grâce à des spécificités institutionnelles dont ils sont les seuls à pouvoir disposer au regard de tous les autres centres d'enseignement du FLE. Mais, comme l'ont montré les trois actions décrites plus haut, les relations entre enseignement et recherche sont largement le fait d'initiatives d'acteurs individuels, enseignants ou enseignants-chercheurs du centre de langue. Il arrive que les innovations apportées par ces individus finissent par donner une identité à l'institution. Tel centre est connu pour ses travaux en phonétique, tel autre pour ses outils numériques. Cette personnalisation comporte cependant deux problèmes liés. D'une part, les réflexions et créations d'outils n'impliquent pas le centre de FLE dans son ensemble, de l'autre, cela fait courir le risque d'absence de pérennisation de l'innovation mise en œuvre. C'est donc bien au niveau de l'interface entre la dimension didactique et la dimension institutionnelle que les choses se jouent pour que convergent l'enseignement du FLE et une recherche-intervention en didactique du FLE.

\section{Références}

Abry, D., Fert, C., Parpette, C., Stauber, J. (2006). ICI 1, méthode de français langue étrangère, Paris : CLE International.

Akrich, M. (2006). Les objets techniques et leurs utilisateurs. De la conception à l'action. Dans M. Akrich, M. Callon et B. Latour. Sociologie de la traduction. Textes fondateurs (p.179-199). Paris : Les Presses Mines.

Akrich, M., Callon, M. \& Latour, B. (1991). L'art de l'intéressement. Dans D. Vinck (coord.). Gestion de la recherche. Nouveaux problèmes, nouveaux outils (p. 4-17). Bruxelles : De Boeck.

Bouchard, R., Parpette, C. et Pochard, J-C. (2005). Le cours magistral et son double, le polycopié : relations et problématique de réception en L2. Cahiers du Français Contemporain, 10, 191-208. 
Bouchard, R. et Parpette, C. (2012). Littéracie universitaire et oralographisme : le cours magistral entre écrit et oral. Pratiques 53-54, 195-210.

Bouchet, K. et Parpette, C. (2017). Dialogisme et réception des cours magistraux en Français langue étrangère. Cahiers de l'ACEDLE, 2. https://journals.openedition.org/rdlc/1852

Campus-FLE ADCUEFE. (s. d.). «Mission». https://www.campus-fle.fr/fr/decouvrircampus-fle/mission/

Castellotti, V. (2013). L'articulation recherche-intervention en didactique des langues : comment (ne pas) en sortir? Dans J.-C Beacco (dir.) Éthique et politique en didactique des langues. Autour de la notion de responsabilité, (p. 74-98). Paris : Didier, Collection Langues et didactique.

Coste D. (2013). La didactique des langues entre pôles d'attraction et lignes de fractures. Dans J.-C. Beacco (dir.) Éthique et politique en didactique des langues. Autour de la notion de responsabilité, (p. 26-73). Paris : Didier, Collection Langues et didactique.

Desgagné, S. (1997). Le concept de recherche collaborative : l'idée d'un rapprochement entre chercheurs universitaires et praticiens enseignants. Revue des sciences de l'éducation, 23(2), 371-393. https://doi.org/10.7202/031921ar

Desgagné, S., Bednarz, N., Lebuis, P., Poirier, L. et Couture, C. (2001). L'approche collaborative de recherche en éducation : un rapport nouveau à établir entre recherche et formation. Revue des sciences de l'éducation, 27(1), 33-64. https://doi.org/10.7202/000305ar

Dufour, S. et Parpette, C. (2017). Le cours magistral : questions d'analyse de discours, questions de didactique. Les carnets du CEDISCOR, Analyse de discours et didactique des/en langues. https://journals.openedition.org/cediscor/1002

Dufour, S. et Parpette, C. (2018). Le français sur objectif spécifique : la notion d'authentique revisitée. ILCEA, 32. https://journals.openedition.org/ilcea/

Goigoux, R. (2017). Associer chercheurs et praticiens à la conception d'outils didactiques ou de dispositifs innovants pour améliorer l'enseignement. Éducation et didactique, 11(3). http://journals.openedition.org/educationdidactique/2872

Grassin, J.-F. (2013). Un réseau social pour le FLE. Quelles perceptions et quels usages de l'apprenant? Recherches en didactique des langues et des cultures, 10(2). https://doi.org/10.4000/rdlc. 1588

Grassin, J.-F. (2017a). Un réseau socio-pédagogique dans une formation en langue : un écran qui reste opaque à l'enseignant? Interfaces numériques, 6(2), 331-349. https://doi.org/10.25965/interfaces-numeriques.2772

Grassin, J.-F. (2016). Un réseau socio-pédagogique pour une formation en français langue étrangère (FLE) : des temporalités nouvelles pour l'enseignement/apprentissage ? Distances et médiations des savoirs. Distance and Mediation of Knowledge, 16. https://doi.org/10.4000/dms.1613

Huver, E. et Charbonneau, D. (2018). CUEFEE de l'université de Tours: profils d'enseignants entre recherche et intervention. Le Français Dans Le Monde, 417, 42.

Olivier de Sardan, J.-P. (2008). La rigueur du qualitatif. Les contraintes empiriques de l'interprétation socio-anthropologique. Louvain-La-Neuve : Academia-Bruylant.

Mangiante, J-M. et Parpette, C. (2004). Le français sur objectif spécifique : de l'analyse des besoins à l'élaboration d'un cours. Grenoble : PUG.

Marcel, J.-F. et Bedin, V. (2018). Contribution à l'élaboration d'un dispositif d'évaluation de la Recherche-Intervention. Phronesis, 7(1), 79-91.

Parpette, C. et Stauber, J. (2014). Réussir ses études d'économie-gestion en français. Grenoble : Presses universitaires de Grenoble.

Paveau, M.-A. (2017). L'Analyse du discours numérique. Dictionnaire des formes et des pratiques. Paris : Hermann. 
Perrenoud, P. (2001). Développer la pratique réflexive dans le métier d'enseignant Professionnalisation et raison pédagogique. Paris : ESF.

Rivens-Mompean, A. (2013). Le Centre de ressources en langues : vers la modélisation du dispositif d'apprentissage. Lille : Presses universitaires du Septentrion.

Schön, D. A. (1993/1983). Le praticien réflexif. A la recherche du savoir caché dans l'agir professionnel. Montréal : Éditions logiques. 